Discrete Comput Geom 26:147-171 (2001)

DOI: $10.1007 / \mathrm{s} 00454-001-0020-4$

\title{
On Quasiperiodic Space Tilings, Inflation, and Dehn Invariants*
}

\author{
O. Ogievetsky ${ }^{1}$ and Z. Papadopolos ${ }^{2}$ \\ ${ }^{1}$ Center of Theoretical Physics, Luminy, \\ 13288 Marseille, France \\ oleg@cpt.univ-mrs.fr \\ ${ }^{2}$ Institut für Theoretische Physik, Universität Magdeburg, \\ PSF 4120, 39016 Magdeburg, Germany \\ zorka.papadopolos@uni-tuebingen.de
}

\begin{abstract}
We introduce Dehn invariants as a useful tool in the study of the inflation of quasiperiodic space tilings. Tilings by "golden tetrahedra" are considered. We discuss how Dehn invariants can be applied to the study of the inflation properties of the six golden tetrahedra. We also use the geometry of the faces of the golden tetrahedra to analyze their inflation properties. We give inflation rules for decorated Mosseri-Sadoc tiles in the projection class of tilings $\mathcal{T}^{\text {(MS) }}$ (the tiles of $\mathcal{T}^{\text {(MS) }}$ are particular packages of the golden tetrahedra). The Dehn invariants of the Mosseri-Sadoc tiles provide two eigenvectors of the inflation matrix with eigenvalues equal to $\tau=(1+\sqrt{5}) / 2$ and $-1 / \tau$, and allow us to reconstruct the inflation matrix uniquely.
\end{abstract}

\section{Introduction}

Existing mathematical models of quasiperiodic tilings of the plane [1]-[3] and threedimensional space [4]-[9] admit an important operation called inflation. Given a tiling of the plane or space by prototiles $\left\{X^{i}\right\}$ from a local isomorphism class of tilings [3] or specie [7], inflation produces another tiling of the class out of the first one, by blowing up the tiles with a factor $\lambda$ ( $\lambda$ is bigger than 1 and called the inflation factor) and substituting the $\lambda$-scaled tiles $X_{(\lambda)}^{i}$ in a particular way by the tiles $X^{j}$ of the original size. Generally, in the process of inflation, the tiles $X^{j}$ are cut into pieces (by hyperplane cuts) and these smaller pieces can then be recombined into the tiles $X_{(\lambda)}^{i}$. The tile $X_{(\lambda)}^{i}$ is made out of

\footnotetext{
* Oleg Ogievetsky was supported by Procope Grant 99082 and is on leave of absence from the Theoretical Department, P. N. Lebedev Physical Institute, Leninsky pr. 53, 117924 Moscow, Russia. Zorka Papadopolos was supported by the Deutsche Forschungsgemeinschaft.
} 
pieces of tiles $X^{j}$ for all $j$. In this paper we discuss tilings of three-dimensional space. Let $M_{j}^{i}$ be the sum of the volumes of the pieces of the tiles of type $X^{j}$, contributing to the covering of $X_{(\lambda)}^{i}$, divided by the volume of $X^{j}$. The dimensionless matrix $M=M_{j}^{i}$ is called the volume inflation matrix.

By its definition, the matrix $M$ has an eigenvector $\vec{v}$ with components $v^{i}=\operatorname{Vol}\left(X^{i}\right)$, the volumes of the tiles. The corresponding eigenvalue is $\lambda^{3}$.

In some cases, the matrix $M$ has rational entries. ${ }^{1}$ Let $\mathbb{Q}[\lambda]$ be the extension of the field $\mathbb{Q}$ of rational numbers by $\lambda$ and let $G=\operatorname{Gal}(\mathbb{Q}[\lambda] / \mathbb{Q})$ be its Galois group. Let $G \lambda=\left\{\lambda_{1}=\lambda, \lambda_{2}, \ldots, \lambda_{k}\right\}$ be the orbit of $\lambda$. Then all the $\lambda_{i}^{3}$ are eigenvalues of the matrix $M$.

In some physically interesting cases, $\lambda$ is a power of the golden mean $\tau=(1+\sqrt{5}) / 2$; the field $\mathbb{Q}[\tau]$ is quadratic and therefore, for rational $M$, volumes can be used to build two eigenvectors (and eigenvalues) of $M$.

One of the questions which we address in this article is an understanding of the geometrical meaning of other eigenvectors of $M$.

We need several standard definitions. One says that two polyhedra, $P_{1}$ and $P_{2}$, are scissor-equivalent (notation: $P_{1} \sim P_{2}$ ) if $P_{1}$ can be cut (by plane cuts) and rebuilt into $P_{2}$.

Assume that there is a function $\mathcal{F}$ which associates an element of some ring to any polyhedron. The function $\mathcal{F}$ is called scissor-invariant if $\mathcal{F}$ enjoys the property $P_{1} \sim P_{2} \Rightarrow \mathcal{F}\left(P_{1}\right)=\mathcal{F}\left(P_{2}\right)$.

A particular case of inflation with a rational inflation matrix is "stone" inflation: the $\lambda$-scaled tiles $X_{(\lambda)}^{i}$ are built of entire tiles $X^{j}$. In this case any scissor-invariant additive function $\mathcal{F}$ allows us to construct an eigenvector $\vec{f}$ of the matrix $M, f^{i}=\mathcal{F}\left(X^{i}\right)$. The comment about the Galois group holds for the vector $\vec{f}$ as well.

It is well known that, starting from dimension 3 , the space of scissor-invariant functions is nontrivial: in addition to the volume, there are also Dehn invariants.

The main aim of this paper is to develop a technique based on Dehn invariants and apply it to the study of inflation rules in dimension 3 . We illustrate our method on two examples: tilings of the space by the golden tetrahedra and by Mosseri-Sadoc tiles.

In Section 2 we give some basic facts about Dehn invariants.

In Section 3 we consider Dehn invariants of golden tetrahedra. We use Dehn invariants as a test for the existence of a stone inflation for golden tetrahedra (Section 3.2). We show that if a Dehn-compatible rational inflation ${ }^{2}$ for golden tetrahedra with the inflation factor $\tau$ exists, then the inflation matrix can be uniquely reconstructed with the help of volumes and Dehn invariants. This unique inflation matrix $M_{\mathrm{gt}}$ turns out to have noninteger entries which show that a stone inflation of golden tetrahedra with the inflation factor $\tau$ cannot exist. However, $M_{\mathrm{gt}}^{3}$, the cube of the matrix $M_{\mathrm{gt}}$, is integer-valued, so we cannot exclude the possibility of a stone inflation for golden tetrahedra with an inflation factor $\tau^{3}$. Note that the existence of the integer (volume) inflation matrix does not guarantee the

\footnotetext{
${ }^{1}$ An example of an exception is the volume inflation matrix of the class of tilings $\mathcal{T}^{*(2 F)}$ icosahedrally projected from the lattice $D_{6}[8]-[10]$, to be discussed later in this paper. By the "icosahedral projection" we mean the icosahedrally invariant projection.

${ }^{2}$ See Section 3.2 for the definition of "Dehn-compatible"; "rational" here means an inflation whose inflation matrix has rational entries.
} 
existence of stone inflation rules. It does not even guarantee the existence of any inflation rules for the tiles. Also we provide an argument that the nonstone inflation for golden tetrahedra found in [9] is not Dehn-compatible.

An alternative proof of the nonexistence of a stone inflation for golden tetrahedra is given in Section 3.3. It is based either on analysis of the irrationalities of areas of faces of the golden tetrahedra or on consideration of the face angles. The analysis in Section 3.3 allows us to show that a stone inflation for golden tetrahedra with an inflation factor $\tau^{k}$, $k=1,2,3, \ldots$, cannot exist for any $k$.

In Section 4.1 we present inflation rules for the decorated Mosseri-Sadoc tiles (they are unions of the golden tetrahedra). We obtain these rules by a local derivation from the inflation rules for the decorated golden tetrahedra (decoration increases the number of tiles: there are eight decorated golden tetrahedra) as the tiles of the projection class $\mathcal{T}^{*(2 F)}[8]-[11]$.

In Section 4.2 we show that the inflation matrix in the case of Mosseri-Sadoc tilings is uniquely reconstructed from the volumes of the prototiles and their Dehn invariants. Also, we explain in Section 4.2 that the inflation matrix for Mosseri-Sadoc tiles is induced by the inflation matrix for golden tetrahedra.

For calculation of the Dehn invariants of the golden tetrahedra we use a ConwayRadin-Sadun theorem (see the Appendix).

\section{Dehn Invariants}

The Dehn invariant of a polyhedron $P$ takes values in a ring $\mathbf{R} \otimes \mathbf{R}_{\pi}$ where $\mathbf{R}_{\pi}$ is the additive group of residues of real numbers modulo $\pi$; the tensor product is over $\mathbf{Z}$, the ring of rational integers. Denote by $l_{i}$ the lengths of edges of $P$. Denote by $\alpha_{i}$ the corresponding lateral angles and by $\bar{\alpha}_{i}$ the residue classes of $\alpha_{i}$ modulo $\pi$. The Dehn invariant, $\mathcal{D}(P)$, of the polyhedron $P$ is equal to

$$
\mathcal{D}(P)=\sum l_{i} \otimes \bar{\alpha}_{i}
$$

with the sum over all edges of $P$.

The Dehn invariant is additive: if a polytope $P$ is a disjoint union of polytopes $P_{\alpha}$, $\alpha=1, \ldots, k$, then

$$
\mathcal{D}(P)=\sum \mathcal{D}\left(P_{\alpha}\right)
$$

Historically, Dehn invariants appeared in solving Hilbert's third problem [12] which asks whether one can calculate the volume of a polyhedron without a limiting procedure. More precisely, given two polyhedra of the same volume, can we cut one and paste the pieces to build another one? Alternatively, is equality of volumes of two polyhedra sufficient for their scissor equivalence?

Dehn [13] has shown that the quantity (1) is scissor-invariant and gave an example of two polyhedra of the same volume but having different Dehn invariants. Thus, equality of Dehn invariants is a necessary condition for scissor equivalence. Later, Sydler [14] has shown that in dimension 3 the equality of volumes and Dehn invariants is also a sufficient condition for scissor equivalence. See [15] for more information on Dehn invariants. 


\section{Inflation of Golden Tetrahedra}

In this section we discuss several aspects of the inflation of golden tetrahedra, not only the inflation of these tiles as the prototiles in the projection class of the tilings $\mathcal{T}^{*(2 F)}$. The projection class of the locally isomorphic tilings $\mathcal{T}^{*(2 F)}$ has been studied in [8]. There, the golden tetrahedra appear through icosahedral projection of the three-dimensional Voronoi cell boundaries of the lattice $D_{6}$. The inflation rules for the tiles in this class have been determined in [9] and [10]. However, it is still an open question whether the class $\mathcal{T}^{*(2 F)}$ is the only class of tilings of the space by golden tetrahedra.

\subsection{Golden Tetrahedra and Their Dehn Invariants}

Golden triangles are triangles with edge lengths 1 and $\tau$ (in some scale) satisfying the condition that not all edges of a triangle are congruent. There are two golden triangles: one with edge lengths $(1,1, \tau)$ and one with edge lengths $(1, \tau, \tau)$. A property of the golden triangles is that edges of each of them can be aligned in the plane parallel to the symmetry axes of a given pentagon.

Golden tetrahedra are tetrahedra with edge lengths 1 and $\tau$ (therefore the faces of golden tetrahedra can be either golden or regular triangles) satisfying the condition that not all faces of a tetrahedron are congruent. Properties of golden tetrahedra are: all edges of golden tetrahedra can be aligned in the space parallel to the twofold symmetry axes of a given icosahedron; the faces of golden tetrahedra which are regular triangles are orthogonal to the threefold symmetry axes of the icosahedron; the faces which are golden triangles are orthogonal to the fivefold symmetry axes of the icosahedron.

There could be seven golden tetrahedra but it turns out that one of them is flat. The six nonflat golden tetrahedra, $G^{*}, F^{*}, A^{*}, B^{*}, D^{*}$, and $C^{*}$, are shown in Fig. 1.

To our knowledge the golden tetrahedra related to the space tilings have been introduced independently by different authors [6], [16], [8].

All the lateral angles of the golden tetrahedra are expressed in terms of four acute $(<\pi / 2)$ angles $\alpha, \beta, \gamma$, and $\delta$ :

$$
\begin{aligned}
& \cos \alpha=\frac{\tau}{\tau+2}=\frac{1}{\sqrt{5}}, \\
& \cos \beta=\frac{\tau+1}{\sqrt{3} \sqrt{\tau+2}}, \\
& \cos \gamma=\frac{\tau+2}{3 \tau}=\frac{\sqrt{5}}{3}, \\
& \cos \delta=\frac{\tau-1}{\sqrt{3} \sqrt{\tau+2}} .
\end{aligned}
$$

The lateral angle $\alpha$ (and $\pi-\alpha$ ) is between the faces in the planes orthogonal to the fivefold symmetry axes of the icosahedron, $\beta$ (and $\pi-\beta$ ) is between the faces in the planes orthogonal to the threefold and fivefold axes, $\gamma$ (and $\pi-\gamma$ ) is between the faces in the planes orthogonal to the threefold axes, and $\delta$ (and $\pi-\delta$ ) is between the faces in the planes orthogonal to the threefold and fivefold axes. 

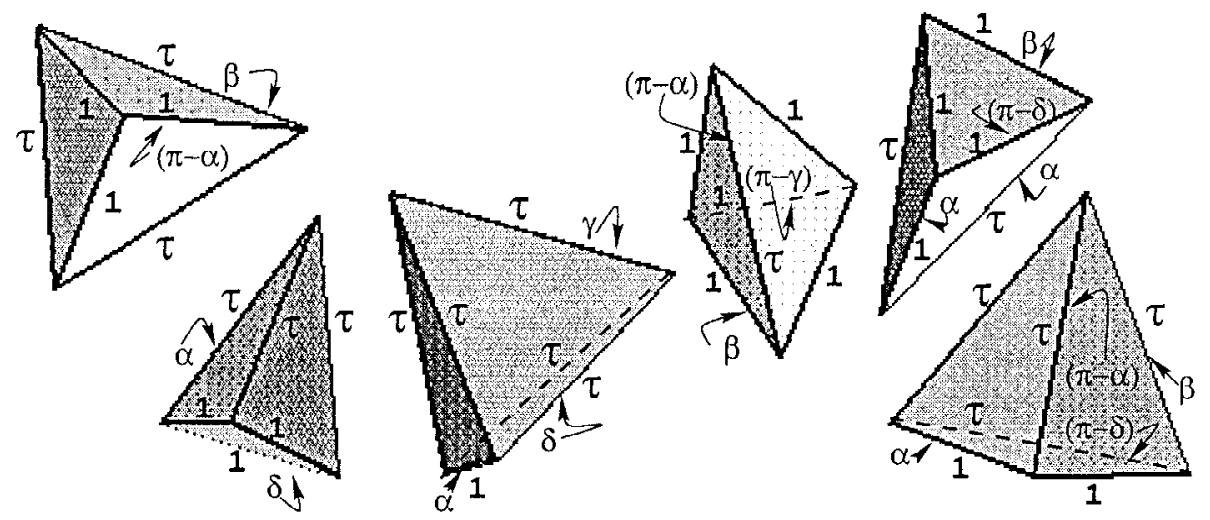

Fig. 1. The tiles of the projection class of the tilings $\mathcal{T}^{*(2 F)}: G^{*}, F^{*}, A^{*}, B^{*}, D^{*}$, and $C^{*}$ (from left to right), the golden tetrahedra. All edges of the tetrahedra are parallel to the twofold symmetry axes of the icosahedron. They are of the standard length (2) (denoted by 1 in the figure) and $\tau$ (2) (denoted by $\tau$ in the Figure), (2) $=\sqrt{2 /(\tau+2)}$. The representative lateral angles are shown. The $\mathbf{Z}_{3}$ rotational symmetry of the tiles $G^{*}$ and $F^{*}$, the $\mathbf{Z}_{2}$ rotational symmetry of the tiles $A^{*}$ and $B^{*}$, and the reflection symmetry of the tiles $D^{*}$ and $C^{*}$ allow us to reconstruct all other lateral angles.

In $\mathbf{R}_{\pi}$ there are linear dependences between the lateral angles $\alpha, \beta, \gamma$, and $\delta$.

\section{Lemma 1.}

$$
\begin{aligned}
\alpha+\gamma+2 \beta & =\pi, \\
\alpha-\gamma+2 \delta & =\pi .
\end{aligned}
$$

Proof. Straightforward.

Therefore, in $\mathbf{R}_{\pi}$ we have the relations

$$
\begin{aligned}
& \bar{\alpha}=-\bar{\beta}-\bar{\delta}, \\
& \bar{\gamma}=-\bar{\beta}+\bar{\delta} .
\end{aligned}
$$

The next step is to prove that there are no more relations: in other words, the images of angles $\beta$ and $\delta$ are independent in $\mathbf{R}_{\pi}$. Because of (6) and (7) it is sufficient to check the independence of $\bar{\alpha}$ and $\bar{\gamma}$.

Lemma 2. The images of angles $\alpha$ and $\gamma$ in $\mathbf{R}_{\pi}$ are independent.

Proof. For notation see the Appendix.

The angles $\alpha$ and $\gamma$ are pure geodetic. One can check that

$$
\alpha=\langle 5\rangle_{1}, \quad \gamma=\frac{\pi}{2}-2\langle 3\rangle_{5} .
$$


The angles $\langle 5\rangle_{1}$ and $\langle 3\rangle_{5}$ are elements of the basis constructed by Conway, Radin, and Sadun. Thus, by the Conway-Radin-Sadun theorem (see the Appendix), the angles $\alpha$ and $\gamma$ are independent.

Calculation of Dehn invariants of the golden tetrahedra is now immediate. We use $\beta$ and $\delta$ as independent angles. We express the Dehn invariants of the golden tetrahedra by the vector $\vec{d}_{\mathrm{gt}}$ :

$$
\vec{d}_{\mathrm{gt}}=\mathcal{D}\left(\begin{array}{c}
A^{*} \\
B^{*} \\
C^{*} \\
D^{*} \\
F^{*} \\
G^{*}
\end{array}\right)=\left(\begin{array}{c}
-\tau-1 \\
\tau+5 \\
3 \tau-2 \\
-2 \tau \\
-3 \tau \\
3 \tau+3
\end{array}\right) \otimes \bar{\beta}+\left(\begin{array}{c}
5 \tau-1 \\
\tau-1 \\
-2 \\
-2 \tau-3 \\
-3 \tau+3 \\
3
\end{array}\right) \otimes \bar{\delta} .
$$

The subscript gt stands for "golden tetrahedra".

The vector $\vec{v}_{\mathrm{gt}}$ of volumes of the golden tetrahedra is

$$
\vec{v}_{\mathrm{gt}}=\mathrm{Vol}\left(\begin{array}{c}
A^{*} \\
B^{*} \\
C^{*} \\
D^{*} \\
F^{*} \\
G^{*}
\end{array}\right)=\frac{1}{12}\left(\begin{array}{c}
2 \tau+1 \\
1 \\
\tau+1 \\
\tau \\
\tau+1 \\
\tau
\end{array}\right)
$$

\subsection{On Inflation of Golden Tetrahedra}

First we show how to use the Dehn invariants as a necessary condition for the existence of stone inflation. By definition, the inflation is "stone" [7] if the inflated tiles are composed of the whole original tiles; in other words, one does not need to cut the original tiles into smaller pieces. In particular, it follows that the volume matrix of a stone inflation has integer entries.

Lemma 1. The golden tetrahedra as prototiles of a space tiling do not admit a stone inflation with an inflation factor $\tau$.

Proof. Assume that a stone inflation exists. Let $M_{\mathrm{gt}}$ be its volume inflation matrix. Since the inflation is stone, the matrix elements of $M_{\mathrm{gt}}$ are rational integers. In particular, $M_{\mathrm{gt}}$ is stable under the action of the Galois group, $\tau \rightarrow-1 / \tau$.

The vector $\vec{v}_{\mathrm{gt}}$ (the vector of volumes of the tiles, (10)) is an eigenvector of $M_{\mathrm{gt}}$ with an eigenvalue $\tau^{3}$.

The additivity of Dehn invariants implies that the vector $\vec{d}_{\mathrm{gt}}$ (the vector of Dehn invariants of the tiles, (9)) is an eigenvector of $M_{\mathrm{gt}}$ with an eigenvalue $\tau$ (the eigenvalue is $\tau$ because Dehn invariants have dimension [length] $]^{1}$ ). Decomposing the vector of Dehn invariants in $\bar{\beta}$ and $\bar{\delta}$ we obtain two eigenvectors of $M_{\mathrm{gt}}$ with the eigenvalue $\tau$. 
Explicitly, we have for the volume vector:

$$
M_{\mathrm{gt}}\left(\begin{array}{c}
2 \tau+1 \\
1 \\
\tau+1 \\
\tau \\
\tau+1 \\
\tau
\end{array}\right)=\left(\begin{array}{c}
8 \tau+5 \\
2 \tau+1 \\
5 \tau+3 \\
3 \tau+2 \\
5 \tau+3 \\
3 \tau+2
\end{array}\right),
$$

for the $\bar{\beta}$-component of the Dehn vector:

$$
M_{\mathrm{gt}}\left(\begin{array}{c}
-\tau-1 \\
\tau+5 \\
3 \tau-2 \\
-2 \tau \\
-3 \tau \\
3 \tau+3
\end{array}\right)=\left(\begin{array}{c}
-2 \tau-1 \\
6 \tau+1 \\
\tau+3 \\
-2 \tau-2 \\
-3 \tau-3 \\
6 \tau+3
\end{array}\right)
$$

and, for the $\bar{\delta}$-component of the Dehn vector:

$$
M_{g t}\left(\begin{array}{c}
5 \tau-1 \\
\tau-1 \\
-2 \\
-2 \tau-3 \\
-3 \tau+3 \\
3
\end{array}\right)=\left(\begin{array}{c}
4 \tau+5 \\
1 \\
-2 \tau \\
-5 \tau-2 \\
-3 \\
3 \tau
\end{array}\right)
$$

The Galois automorphism $\tau \rightarrow-1 / \tau$ produces three more eigenvectors of $M_{\mathrm{gt}}$. Since the entries of $M_{\mathrm{gt}}$ are integer, to use the Galois automorphism is the same as to decompose the vector equalities (11), (12), and (13) in the powers of $\tau$ (i.e., consider the $\tau^{0}$ and $\tau^{1}$-components of (11), (12), and (13)). Writing all the columns together we obtain the matrix equality:

$$
M_{\mathrm{gt}}\left(\begin{array}{rrrrrr}
2 & 1 & -1 & -1 & 5 & -1 \\
0 & 1 & 1 & 5 & 1 & -1 \\
1 & 1 & 3 & -2 & 0 & -2 \\
1 & 0 & -2 & 0 & -2 & -3 \\
1 & 1 & -3 & 0 & -3 & 3 \\
1 & 0 & 3 & 3 & 0 & 3
\end{array}\right)=\left(\begin{array}{rrrrrr}
8 & 5 & -2 & -1 & 4 & 5 \\
2 & 1 & 6 & 1 & 0 & 1 \\
5 & 3 & 1 & 3 & -2 & 0 \\
3 & 2 & -2 & -2 & -5 & -2 \\
5 & 3 & -3 & -3 & 0 & -3 \\
3 & 2 & 6 & 3 & 3 & 0
\end{array}\right) .
$$

The matrix $M_{\mathrm{gt}}$ is acting on a $6 \times 6$ matrix whose first column is the $\tau^{1}$-component of (11), the second column is the $\tau^{0}$-component of (11), the third and fourth columns are the $\tau^{1}$ - and $\tau^{0}$-components of (12), and the fifth and sixth columns are the $\tau^{1}$ - and $\tau^{0}$-components of (13).

Equation (14) is the matrix equation for the matrix $M_{\mathrm{gt}}$. We found the complete basis of eigenvectors, therefore the solution is unique and we find

$$
M_{\mathrm{gt}}=\left(\begin{array}{cccccc}
2 & 0 & 1 & 0 & 2 & 1 \\
0 & 0 & 1 & 0 & 0 & 1 \\
\frac{1}{2} & \frac{1}{2} & 1 & 1 & 1 & 1 \\
0 & 0 & 1 & 1 & 1 & 0 \\
1 & 0 & 1 & 1 & 1 & 0 \\
\frac{1}{2} & \frac{1}{2} & 1 & 0 & 0 & 1
\end{array}\right)
$$


The matrix entries of $M_{\mathrm{gt}}$ are not integers therefore a stone inflation with the inflation factor $\tau$ cannot exist.

We actually proved more. To give a precise formulation we need a new definition.

Consider an inflation with an inflation factor $\lambda$. Assume that $\lambda$-scaled tiles $X_{(\lambda)}^{i}$ are not built of entire tiles $X^{j}$ (thus, the inflation is not necessarily stone). Consider a portion of a tile $X^{j}$, say $Y$, which appears in the $\lambda$-scaled tile $X_{(\lambda)}^{i}$. Let $\mathcal{F}$ be an additive scissorinvariant function.

Definition. We say that the inflation is $\mathcal{F}$-compatible if $\mathcal{F}(Y)=\mu \mathcal{F}\left(X^{j}\right)$, where the number $\mu$ is defined by $\operatorname{Vol}(Y)=\mu \operatorname{Vol}\left(X^{j}\right)$, for all portions $Y$ of tiles $X^{j}$ which appear in all scaled tiles $X_{(\lambda)}^{i}$.

For an $\mathcal{F}$-compatible inflation, the vector $\mathcal{F}\left(X^{i}\right)$ is an eigenvector of the inflation matrix. A stone inflation is $\mathcal{F}$-compatible for any $\mathcal{F}$. If the inflation is compatible with the Dehn invariant we say, for short, that it is Dehn-compatible.

The proof of the Lemma 1 of this section implies the following statement.

Lemma 1'. If a Dehn-compatible inflation (for the golden tetrahedra) with a rational inflation matrix existed, then the inflation matrix would necessarily be equal to (15).

In other words, having assumed that the inflation matrix is rational we could reconstruct it uniquely. This happened because of a coincidence: $2 \times 3=6$. Here 2 is the order of the Galois group, 3 is the number of independent invariants (the volume and the two Dehn invariants), while 6 is the number of tiles. Due to this coincidence we obtained the matrix equation for $M_{\mathrm{gt}}$ admitting a unique solution. We do not have a good explanation for this coincidence.

An inflation, with the inflation factor $\tau$ for the golden tetrahedra as the prototiles of the projection class of the tilings $\mathcal{T}^{*(2 F)}$ (obtained by the icosahedrally invariant projection from the $D_{6}$ lattice), has been found in [9] and [10]. There, one has to divide the tiles $C^{*}$ and $G^{*}$, each into two subtypes: "blue" and "red", and these subtypes inflate differently. Therefore, the number of tiles becomes 8 . The volume inflation matrix $M_{\mathcal{T}^{*(2 F)}}$ is equal to

$$
\left(\begin{array}{cccccccc}
11 \tau-16 & 0 & 2 \tau-2 & 2 \tau-3 & 0 & 9 \tau-13 & \tau-1 & 3 \tau-4 \\
0 & 0 & 0 & 1 & 0 & 0 & 0 & 1 \\
-2 \tau+4 & 1 & 0 & -\tau+2 & 1 & -\tau+3 & 0 & -\tau+2 \\
-9 \tau+15 & 0 & -2 \tau+4 & -\tau+2 & 1 & -8 \tau+14 & -\tau+2 & -2 \tau+4 \\
0 & 0 & 0 & 1 & 1 & 1 & 0 & 0 \\
1 & 0 & 1 & 0 & 1 & 1 & 0 & 0 \\
-2 \tau+4 & 1 & 0 & -\tau+2 & 0 & -\tau+2 & 0 & -\tau+2 \\
-9 \tau+15 & 0 & -2 \tau+4 & -\tau+2 & 0 & -8 \tau+13 & -\tau+2 & -2 \tau+4
\end{array}\right)
$$

in the following ordering of the tiles: $A^{*}, B^{*}, C^{* \mathrm{~b}}, C^{* \mathrm{r}}, D^{*}, F^{*}, G^{* \mathrm{~b}}$, and $G^{* \mathrm{r}}$. The superscripts "b" and " $r$ " denote the "blue" and "red" variants of tiles, respectively.

It is interesting to note that: (i) for the tiles $B^{*}, D^{*}$, and $F^{*}$ the inflation matrices $M_{\mathrm{gt}}$ and $M_{\mathcal{T}^{*(2 F)}}$ give the same results (up to colors); (ii) noninteger entries in (15) appear 
exactly in the columns corresponding to the tiles $C^{*}$ and $G^{*}$ - the tiles which are getting blue and red colors in the inflation with matrix (16).

Remark. The matrix $M_{\mathcal{T}^{*(2 F)}}$ has an eigenvalue $\tau$ (with multiplicity 1 ) and the corresponding eigenvector is

$$
\vec{v}_{\tau}=\left(\begin{array}{c}
5 \tau+2 \\
-7 \tau+11 \\
4 \tau-9 \\
4 \tau-10 \\
-6 \tau+1 \\
-3 \tau+3 \\
4 \\
3
\end{array}\right) .
$$

It cannot be proportional to the vector of the Dehn invariants of the tiles since the components of $\vec{v}_{\tau}$ corresponding to $C^{* \mathrm{~b}}$ and $C^{* \mathrm{r}}$ (as well as to $G^{* \mathrm{~b}}$ and $G^{* \mathrm{r}}$ ) are different, although geometrically the tiles $C^{* \mathrm{~b}}$ and $C^{* \mathrm{r}}$ (respectively, $G^{* \mathrm{~b}}$ and $G^{* \mathrm{r}}$ ) are identical. Moreover, there are two independent Dehn invariants for the golden tetrahedra while the multiplicity of the eigenvalue $\tau$ of the matrix $M_{\mathcal{T}^{*(2 F)}}$ is 1 . This shows that the inflation of $\mathcal{T}^{*(2 F)}$ is not Dehn-compatible.

Lemma 1 of this section shows that a stone inflation with the inflation factor $\tau$ is impossible. We could however try to construct a hypothetic volume inflation matrix with an inflation factor $\tau^{k}$ with integer positive $k, k>1$. As in the proof of Lemma 1, the volume vectors and the vectors of Dehn invariants fix the inflation matrix uniquely: the only possible inflation matrix with the inflation factor $\tau^{k}$ is the matrix $M_{\mathrm{gt}}^{k}$. It turns out that there are powers of the matrix $M_{\mathrm{gt}}$ which are integer-valued.

Lemma 2. The matrix $M_{\mathrm{gt}}^{k}$ has integer entries if and only if $k$ is divisible by 3.

Proof. A direct calculation gives

$$
M_{\mathrm{gt}}^{2}=\left(\begin{array}{cccccc}
7 & 1 & 6 & 3 & 7 & 4 \\
1 & 1 & 2 & 2 & 1 & 2 \\
3 & 1 & 5 & 3 & 4 & 3 \\
\frac{3}{2} & \frac{1}{2} & 3 & 3 & 3 & 1 \\
\frac{7}{2} & \frac{1}{2} & 4 & 3 & 5 & 2 \\
2 & 1 & 3 & 1 & 2 & 3
\end{array}\right)
$$

and

$$
M_{\mathrm{gt}}^{3}=\left(\begin{array}{rrrrrr}
26 & 5 & 28 & 16 & 30 & 18 \\
5 & 2 & 8 & 4 & 6 & 6 \\
14 & 4 & 19 & 12 & 18 & 12 \\
8 & 2 & 12 & 9 & 12 & 6 \\
15 & 3 & 18 & 12 & 19 & 10 \\
9 & 3 & 12 & 6 & 10 & 9
\end{array}\right)
$$


Thus, $M_{\mathrm{gt}}^{3}$ is an integer-valued matrix and therefore the matrices $M_{\mathrm{gt}}^{3 k}$ are integer-valued as well.

It is left to prove that if $n$ is not a multiple of 3 , then $M_{\mathrm{gt}}^{n}$ is not integer-valued.

By construction, the eigenvalues of $M_{\mathrm{gt}}$ are

$$
\tau^{3},\left(-\tau^{-3}\right), \tau, \text { and }\left(-\tau^{-1}\right)
$$

Therefore, the minimal polynomial for $M_{\mathrm{gt}}$ is

$$
\chi(x)=x^{4}-5 x^{3}+2 x^{2}+5 x+1,
$$

$\chi\left(M_{g t}\right)=0$.

A straightforward check shows that if

$$
x^{4}=5 x^{3}-2 x^{2}-5 x-1
$$

then

$$
x^{n}=a_{n} x^{3}+b_{n} x^{2}+c_{n} x+d_{n}
$$

with

$$
a_{n}=\frac{1}{3}\left(\frac{f_{3(n-1)}}{2}-f_{n-1}\right)
$$

and

$$
\begin{aligned}
b_{n} & =a_{n+1}-a_{n}, \\
c_{n} & =-a_{n+1}+3 a_{n}+f_{n}, \\
d_{n} & =-a_{n+1}+4 a_{n}+f_{n-1} .
\end{aligned}
$$

Here $\left\{f_{n}\right\}$ are Fibonacci numbers defined by $f_{0}=0, f_{1}=1$, and $f_{n+1}=f_{n}+f_{n-1}$.

Therefore,

$$
M_{\mathrm{gt}}^{n}=a_{n} M_{\mathrm{gt}}^{3}+b_{n} M_{\mathrm{gt}}^{2}+c_{n} M_{\mathrm{gt}}+d_{n} \mathbf{I d},
$$

where Id is the unit matrix.

The numbers $a_{n}, b_{n}, c_{n}$, and $d_{n}$ are integer. The matrices $M_{\mathrm{gt}}^{3}$ and Id have integer entries. The matrices $M_{\mathrm{gt}}$ and $M_{\mathrm{gt}}^{2}$ have-at different places-rational entries with the denominator 2. Therefore, the matrix $M_{\mathrm{gt}}^{n}$ has integer entries if and only if the integers $b_{n}$ and $c_{n}$ are even, which means that

$$
a_{n+1} \equiv a_{n} \quad(\bmod 2)
$$

and

$$
-a_{n+1}+3 a_{n}+f_{n} \equiv 0 \quad(\bmod 2)
$$

Substitution of $(27)$ into $(28)$ gives $f_{n} \equiv 0(\bmod 2)$. It is well known that $f_{n}$ is even if and only if $n$ is a multiple of 3 (see, e.g., Chapter 6 of [17]). 
The proof above gives a useful explicit expression (26) for the powers of the matrix $M_{\mathrm{gt}}$. However, the statement of the lemma requires neither knowledge of the eigenvalues of $M_{\mathrm{gt}}$ nor the properties of the Fibonacci numbers:

Second proof. ${ }^{3} \quad$ The matrix $M_{\mathrm{gt}}^{3}$ is integer-valued and its determinant is equal to (-1). Therefore, $M_{\mathrm{gt}}^{-3}$ is integer-valued as well. Suppose that $M_{\mathrm{gt}}^{3 n+j}$ (with $j \in\{1,2\}$ ) is integervalued. Then $M_{\mathrm{gt}}^{j}=M_{\mathrm{gt}}^{-3 n} M_{\mathrm{gt}}^{3 n+j}$ would be integer-valued, which is a contradiction (see (15) and (18)).

To conclude: with the help of the Dehn invariants one is able to show that a stone inflation with the inflation factor $\tau$ is impossible. However, one cannot exclude a stone inflation with the inflation factor $\tau^{3}$.

In the next section we show, using a different method, that a stone inflation with the inflation factor $\tau^{3}$ is impossible as well.

\subsection{Faces of Golden Tetrahedra}

Lemma 1 proved in Section 3.2 shows that a stone inflation with the inflation factor $\tau$ is impossible due to the scissor-invariants of the tiles- the volumes and the Dehn invariants.

Here we give another argument showing the impossibility of a stone inflation. This argument uses the geometry of faces of the tiles.

More precisely, using Dehn invariants amounts to analyzing irrationalities in the lateral angles of the golden tetrahedra. Now we analyze irrationalities in the areas of the faces of the golden tetrahedra.

The faces of the golden tetrahedra are golden and regular triangles.

Denote the regular triangle, with edge length 1 , by $\Delta_{\mathrm{r}}$, the acute golden triangle (with edge lengths $\tau, \tau$, and 1 ) by $\Delta_{\mathrm{a}}$, and the obtuse golden triangle (with edge lengths $\tau, 1$, and 1) by $\Delta_{0}$.

For an arbitrary polygon $\Pi$, a notation $\tau^{-k} \Pi$ means the polygon $\Pi$ scaled by $\tau^{-k}$. Also, for a polygon $\Pi$, denote a set of polygons $\left\{\tau^{-k} \Pi, k=1,2,3, \ldots\right\}$ by $\tau^{-} \Pi$.

The areas $A(\Delta)$ of the triangles are

$$
\begin{aligned}
& A_{\mathrm{r}} \equiv A\left(\Delta_{\mathrm{r}}\right)=\frac{\sqrt{3}}{4}, \\
& A_{\mathrm{o}} \equiv A\left(\Delta_{\mathrm{o}}\right)=\frac{\sqrt{\tau+2}}{4}, \\
& A_{\mathrm{a}} \equiv A\left(\Delta_{\mathrm{a}}\right)=\frac{\tau \sqrt{\tau+2}}{4} .
\end{aligned}
$$

The $\sqrt{ }$ always denotes the positive branch of the square root.

It is interesting to note that the irrationalities in the areas of the faces are exactly the same as in the trigonometric functions of the lateral angles (see (3)): $\sqrt{3}, \tau$, and $\sqrt{\tau+2}$.

\footnotetext{
${ }^{3}$ This proof was proposed by L. Danzer.
} 
We first prove an intuitively obvious technical lemma which shows that irrationalities expressing the areas (29) are different.

Lemma 1. The irrationalities $\sqrt{3}$ and $\sqrt{\tau+2}$ are independent over the field $\mathbb{Q}[\tau]$.

Proof. The number $\rho=\sqrt{\tau+2}$ satisfies an equation $f(\rho)=0$ where

$$
f(x)=x^{4}-5 x^{2}+5 .
$$

By the Eisenstein criterion (see, e.g., Chapter 3 of [18]), the polynomial $f$ is irreducible over $\mathbb{Q}$. Moreover, $f$ splits in $\mathbb{Q}[\rho]$ : its roots are

$$
\pm \sqrt{\tau+2} \text { and } \pm \sqrt{3-\tau}
$$

The irrationality $\sqrt{3-\tau}$ belongs to the field $\mathbb{Q}[\rho]$ : one has

$$
\sqrt{3-\tau}=\tau^{-1} \sqrt{\tau+2} \in \mathbb{Q}[\rho] .
$$

A splitting field of any polynomial is a Galois extension [18, Chapter 4]. Therefore, the field $\mathbb{Q}[\rho]$ —as the splitting field of the polynomial $f$-is the Galois extension of $\mathbb{Q}$.

The automorphism group $\operatorname{Gal}(\mathbb{Q}[\rho] / \mathbb{Q})$ is isomorphic to the cyclic group $\mathbf{Z}_{4}$, with the generator $\sigma$,

$$
\sigma: \sqrt{\tau+2} \rightarrow \sqrt{3-\tau}
$$

In a basis $1, \sqrt{\tau+2}, \tau$, and $\sqrt{3-\tau}$ of $\mathbb{Q}[\rho]$ over $\mathbb{Q}$, the action of $\sigma$ on the other elements of the basis is given by

$$
\sigma: \tau \rightarrow-\tau^{-1} \text { and } \sigma: \sqrt{3-\tau} \rightarrow-\sqrt{\tau+2} .
$$

Hence, $\sigma^{4}=1$.

By the Fundamental Theorem of Galois Theory (see, e.g., Chapter 4 of [18]), a quadratic extension of $\mathbb{Q}$ between $\mathbb{Q}$ and $\mathbb{Q}[\rho]$ can be only the fixed field of $\sigma^{2}$ which is $\mathbb{Q}[\tau]$.

In particular, $\sqrt{3} \notin \mathbb{Q}[\rho]$ (since, clearly, $\sqrt{3} \notin \mathbb{Q}[\tau]$ ).

Remark. It is also easy to prove in an elementary way that the equation $x^{2}=3$ does not have solutions in $\mathbb{Q}[\rho]$.

Corollary 1. The field $\mathbb{Q}[\rho, \sqrt{3}]$ admits an automorphism $\varphi$ which satisfies:

1. $\varphi: \sqrt{3} \rightarrow-\sqrt{3}$;

2. the fixed field of $\varphi$ is $\mathbb{Q}[\rho]$.

Proof. It follows from Lemma 1 of this section that the field $\mathbb{Q}[\rho, \sqrt{3}]$ is a quadratic extension of the field $\mathbb{Q}[\rho]$. In characteristic 0 , any quadratic extension is Galois [18, Chapter 4]. This immediately implies the existence of the automorphism $\varphi$. 
We now apply these algebraic preliminaries to the analysis of a stone inflation.

If a stone inflation existed, the faces of inflated tiles would be covered by the faces of the original tiles.

\section{Lemma 2.}

1. Assume that a regular triangle $\Delta_{\mathrm{r}}$ is covered by a finite (interior)-disjoint union of regular triangles from $\tau^{-} \Delta_{\mathrm{r}}$ and golden triangles from $\tau^{-} \Delta_{\mathrm{a}}$ and $\tau^{-} \Delta_{\mathrm{o}}$. Then the golden triangles are absent in the covering. In other words, a regular triangle can be covered by regular triangles only.

2. Similarly, the golden triangles can be covered by the golden triangles only, the regular triangles must be absent in the covering.

Proof. Suppose that the triangle $\Delta_{\mathrm{r}}$ is covered by a finite union of triangles from $\tau^{-} \Delta_{\mathrm{r}}$, $\tau^{-} \Delta_{\mathrm{a}}$, and $\tau^{-} \Delta_{\mathrm{o}}$. Then for the areas we have

$$
A_{\mathrm{r}}=p_{1}\left(\tau^{-2}\right) A_{\mathrm{r}}+p_{2}\left(\tau^{-2}\right) A_{\mathrm{a}}+p_{3}\left(\tau^{-2}\right) A_{\mathrm{o}},
$$

where $p_{1}, p_{2}$, and $p_{3}$ are polynomials with nonnegative integer coefficients and the polynomial $p_{1}$ does not have a constant term.

Let $X=\sqrt{3}\left(1-p_{1}\left(\tau^{-2}\right)\right)$ and $Y=p_{2}\left(\tau^{-2}\right) \tau \sqrt{\tau+2}+p_{3}\left(\tau^{-2}\right) \sqrt{\tau+2}$. The equality (35) is equivalent to $X=Y$.

Applying the automorphism $\varphi$ (Corollary 1 and Lemma 1 of this section) to the equality $X=Y$ we find $(-X)=Y$ and it follows that $X=0$ and $Y=0$ separately. Since each term in the expressions $p_{2}\left(\tau^{-2}\right) A_{\mathrm{a}}$ and $p_{3}\left(\tau^{-2}\right) A_{\mathrm{o}}$ is nonnegative, the equality $Y=0$ implies that the polynomials $p_{2}(x)$ and $p_{3}(x)$ are identically zero. This means that the golden triangles are absent.

The considerations with coverings of the golden triangles are analogous.

There is another argument which uses consideration of angles instead of consideration of areas and which shows that a stronger statement is valid, one does not need to require that smaller triangles are $\tau^{-k}$ scaled.

\section{Lemma $2^{\prime}$.}

1. Assume that a regular triangle $\Delta_{\mathrm{r}}$ is covered by a finite (interior)-disjoint union of regular triangles and golden triangles. Then the golden triangles are absent in the covering.

2. Similarly, the golden triangles can be covered by the golden triangles only.

Proof. Consider a (connected) polygon $\Pi$ on the plane. Suppose that $\Pi$ is a finite disjoint union of regular and golden triangles. Let $\left\{A_{i}\right\}$ be a set of vertices of all the triangles. Choose a straight line $l$ which is not parallel to any of the straight lines connecting vertices $A_{i}$ and $A_{j}$ for all pairs of different $i$ and $j$. Then, when the line $l$ moves from infinity toward $\Pi$, it meets no more than one vertex at each instance.

Assume that $\Pi$ is a regular triangle, $\Pi=\Delta_{\mathrm{r}}$. Assume that $\Pi$ is covered by regular and golden triangles and golden triangles are present in the covering. There is a first 
instance when the line $l$ meets a vertex, say $A$, of a golden triangle. The total angle $\alpha$ at the vertex $A$ in the covering can take three different values: $60^{\circ}$ if $A$ is the vertex of $\Delta_{\mathrm{r}}$ itself; $180^{\circ}$ if $A$ is inside an edge of $\Delta_{\mathrm{r}} ; 360^{\circ}$ if $A$ is in the interior of $\Delta_{\mathrm{r}}$.

The angles of golden triangles are multiples of $36^{\circ}$. Therefore we have

$$
\alpha=m \cdot 36^{\circ}+n \cdot 60^{\circ} .
$$

By assumption, there is a golden triangle with the vertex $A$. Thus, $m \geq 1$. All golden triangles are strictly on one side of the line $l$, therefore $m \cdot 36^{\circ}<180^{\circ}$ or $m<5$.

It is immediate to see that (36) does not admit solutions with $m \in\{1,2,3,4\}$ when $\alpha \in\left\{60^{\circ}, 180^{\circ}, 360^{\circ}\right\}$. This finishes the proof of part 1 of the lemma.

Similarly, if $\Pi$ is a golden triangle, let $A$ be a first vertex of a regular triangle which meets the line $l$. Then we have (36) with $n \in\{1,2\}$ and $\alpha \in\left\{36^{\circ}, 72^{\circ}, 108^{\circ}, 180^{\circ}, 360^{\circ}\right\}$. Again, there are no solutions which proves part 2 of the lemma.

To prove the nonexistence of a stone inflation we consider coverings of the regular triangle.

We prove that a regular triangle with an edge of length $\tau^{k}$ cannot be covered by regular triangles with edge lengths $\tau^{i}, i=0, \ldots, k-1$. This implies that there is no stone inflation with the inflation factor $\tau^{k}$ for any $k$.

In fact, the same arguments can be applied to coverings of any polygon $\Pi$ by $\tau^{k}$ smaller copies of the same polygon.

Consider an arbitrary polygon $\Pi$. Suppose that the polygon $\tau^{k} \Pi$ is divided into a finite (interior)-disjoint union of polygons $\tau^{i} \Pi$ with $i=0, \ldots, k-1$. Consider such division with a smallest possible $k$. Then a polygon $\Pi=\tau^{0} \Pi$ is necessarily present—otherwise, rescaling by $1 / \tau$ we would obtain the division of the polygon $\tau^{k-1} \Pi$, contradicting the minimality of $k$.

Denote by $\alpha_{i}$ the number of polygons $\tau^{i} \Pi$. We have $\alpha_{i} \geq 0$ for $i=1, \ldots, k-1$ and $\alpha_{0}>0$. Put $\omega=\tau^{2}$. From the area under consideration it follows that

$$
\omega^{k}=\alpha_{k-1} \omega^{k-1}+\cdots+\alpha_{0} .
$$

It is this statement which leads to a contradiction.

Lemma 3. The number $\omega$ cannot satisfy an equation

$$
\omega^{k}-\alpha_{k-1} \omega^{k-1}-\cdots-\alpha_{0}=0,
$$

where $\alpha_{1}, \ldots, \alpha_{k-1}$ are nonnegative integer numbers and $\alpha_{0}$ is a positive integer number.

Proof. The minimal equation (over $\mathbf{Z})$ for $\omega=(3+\sqrt{5}) / 2$ is

$$
\omega^{2}-3 \omega+1=0 .
$$

Let $p(x)=x^{k}-\alpha_{k-1} x^{k-1}-\cdots-\alpha_{0}$. Assume that $p(\omega)=0$. This means that one can divide $p(x)$ by $x^{2}-3 x+1$ :

$$
p(x)=\left(x^{k-2}+\beta_{k-3} x^{k-3}+\cdots+\beta_{0}\right)\left(x^{2}-3 x+1\right) .
$$


Collecting coefficients in powers of $x$ we obtain the following system:

$$
\begin{aligned}
-\alpha_{k-1} & =-3+\beta_{k-3}, \\
-\alpha_{k-2} & =1-3 \beta_{k-3}+\beta_{k-4}, \\
-\alpha_{k-3} & =\beta_{k-3}-3 \beta_{k-4}+\beta_{k-5}, \\
& \vdots \\
-\alpha_{2} & =\beta_{2}-3 \beta_{1}+\beta_{0}, \\
-\alpha_{1} & =\beta_{1}-3 \beta_{0}, \\
-\alpha_{0} & =\beta_{0} .
\end{aligned}
$$

Let $\psi_{n}=f_{2 n+2}$ where $f_{n}$ are Fibonacci numbers. Then we have $\psi_{0}=1, \psi_{1}=3$, and

$$
\psi_{n+1}=3 \psi_{n}-\psi_{n-1} .
$$

Let $S=\alpha_{k-1} \psi_{0}+\alpha_{k-2} \psi_{1}+\cdots+\alpha_{0} \psi_{k-1}$.

Substituting expressions for $\alpha_{i}$ from (42) one finds that due to (42) the terms with $\psi_{i}$ for $i>1$ cancel and one is left with

$$
S=-3 \psi_{0}+\psi_{1} \equiv-3+3=0,
$$

which is impossible since all $\psi_{i}$ are positive, $\alpha_{i}$ are nonnegative, and $\alpha_{0}$ is positive.

Although we do not need it, a stronger statement holds.

Lemma 3'. The number $\omega$ cannot satisfy (38) with $\alpha_{1}, \ldots, \alpha_{k-2}$ nonnegative rational numbers, $\alpha_{k-1}$ a rational number, and $\alpha_{0}$ a positive rational number.

Remark. L. Danzer has drawn our attention to the fact that the lemma follows immediately from Descartes' rule of signs: the number of positive roots of a polynomial $f(x)$ (counted with multiplicities) equals the number $C$ of changes of signs in the system of coefficients of the polynomial $f(x)$ or less than $C$ by an even number (see, e.g., [19]).

Indeed, there is precisely one change of sign in (38) (no condition on $\alpha_{k-1}$ is needed). Therefore (38) cannot have more than one positive root. Nonetheless both $\omega$ and its algebraic conjugate $\omega^{*}=\tau^{-2}=\omega-\sqrt{5}$ are positive and satisfy (38) (since the coefficients are rational).

However, using Fibonacci numbers, one can give a proof, independent of the rule of signs.

Proof. Consider a combination

$$
S^{\prime}=\alpha_{k-2} \psi_{0}+\alpha_{k-3} \psi_{1}+\cdots+\alpha_{2} \psi_{k-4}+\alpha_{1} \psi_{k-3} .
$$

Substituting expressions (42) for $\alpha_{i}$ one finds $S^{\prime}=1-\psi_{k-2} \beta_{0}$. The combination (44) is obviously nonpositive, thus $1-\psi_{k-2} \beta_{0} \leq 0$ and it follows that $\beta_{0}$ is positive. However, by the last line in (42), $\beta_{0}$ is negative, a contradiction. 
As we have seen, Lemma 3 implies the following statement.

Corollary 2. A regular triangle $\Delta_{\mathrm{r}}$ cannot be covered by regular triangles $\tau^{-k} \Delta_{\mathrm{r}}$, $k=1, \ldots, N$ for any $N$.

With these preliminaries we are now prepared to show that a stone inflation for the golden tetrahedra is impossible.

Proposition. For the golden tetrahedra, a stone inflation with the inflation factor $\tau^{k}$, with an arbitrary positive integer $k$, does not exist.

Proof. As was said above, the existence of a stone inflation implies that the faces of the inflated tiles can be covered by the faces of the tiles of the original size. In particular, a face which is an inflated regular triangle, would be covered by regular and golden triangles.

Lemma 2 of this section shows that the golden triangles cannot appear in such covering. Therefore, the inflated regular triangle can be covered by regular triangles onlywhich is impossible by Corollary 2 and Lemma 3.

This contradiction shows that a stone inflation does not exist.

Remark. The known tilings $\mathcal{T}^{*(2 F)}$ have the following property. The golden tetrahedra in the tiling of the space have their edges parallel to the twofold symmetry axes of the icosahedron ("the long range orientational order"). The faces of the tiles which are regular triangles are all located in the planes perpendicular to the threefold symmetry axes of the icosahedron. However, the golden triangles are all perpendicular to the fivefold symmetry axes. Therefore, if a stone inflation for the tilings $\mathcal{T}^{*(2 F)}$ existed, the regular triangles could be covered only by the smaller regular triangles due to the orientation of the faces. In this case we do not need Lemmas 1 and 2 of this section.

We stress again that the existence of "rational" inflation rules for the golden tetrahedra (equation (15)) is hypothetic because in our algebraic approach we do not impose any restriction on the orientations of the tiles in the tiling of the three-dimensional space.

The logic used in this section gives additional motivation to consider minimal packages of the golden tetrahedra in which the regular faces are all hidden (see Section 4).

\section{Mosseri-Sadoc Tiles}

The five prototiles $a, m, r, z$, and $s$ of the projection class of the tilings $\mathcal{T}^{\text {(MS) }}$ (see [20]) are shown in Fig. 2. The tiles $r$ and $m$ in $\mathcal{T}^{(\mathrm{MS})}$ always appear together as a tile $h, h=r \cup m$, see Fig. 3. The prototiles $z, h, s$, and $a$ are of the same shape as the prototiles of the inflation class of the tilings introduced by Sadoc and Mosseri [6], and we call them the Mosseri-Sadoc tiles. The tiles $a, m, r, z$, and $s$ are composed of the golden tetrahedra [6], [20], as shown in Fig. 4, in such a way that the regular triangles of the golden tetrahedra are all hidden [20]. Hence, the faces of the composed tiles $a, m$, 

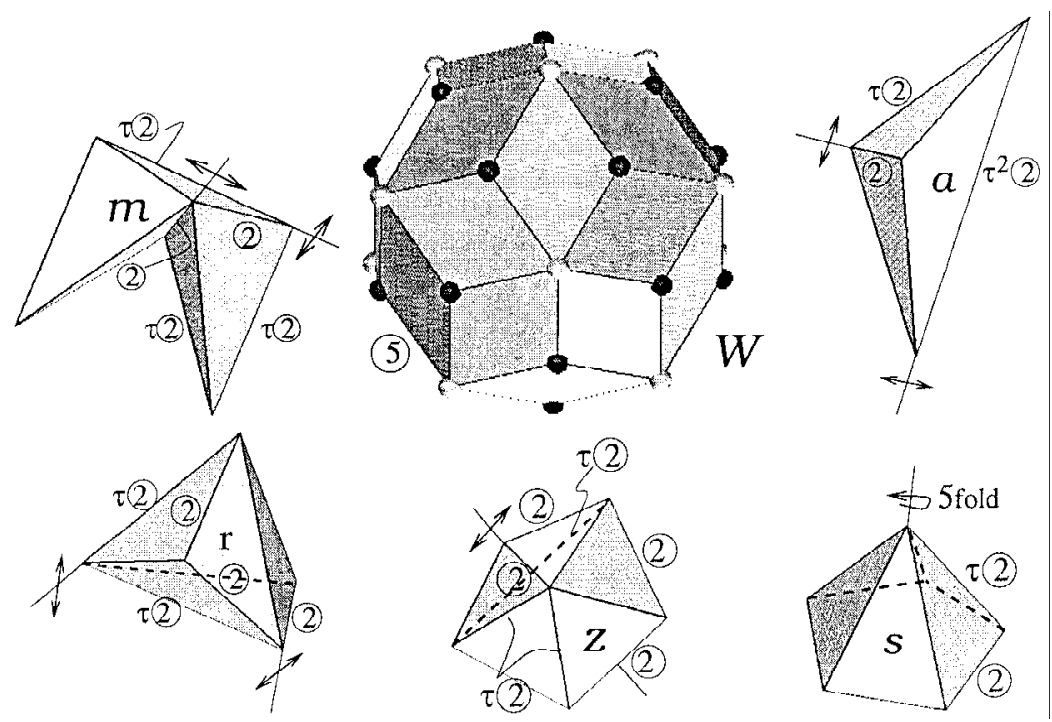

Fig. 2. The outer shape of the "window" $W\left(=V_{\perp}\right)$ of the projection class of the tilings $\mathcal{T}^{(\mathrm{MS})}$ in $\mathbb{E}_{\perp}$. It is the triacontahedron with an edge length $(5)=1 / \sqrt{2}$, the standard length parallel to the fivefold symmetry axes of the icosahedron. The figure shows the tiles $a, r, m, s$, and $z$ in $\mathbb{E}_{\|}$. The symmetries of the tiles and the representative lengths of edges are marked. In this paper the standard length $(2)=\sqrt{2 /(\tau+2)}$ is set to 1 .

$r, z$, and $s$ are golden triangles only. The same is true for the Mosseri-Sadoc tiles $z, h$, $s$, and $a$.

Using the additivity of Dehn invariants one finds the vector of Dehn invariants for the Mosseri-Sadoc tiles:

$$
\vec{d}_{\mathrm{MS}}=\mathcal{D}\left(\begin{array}{l}
z \\
h \\
s \\
a
\end{array}\right)=-5\left(\begin{array}{c}
\tau \\
2 \\
\tau-1 \\
-\tau
\end{array}\right) \otimes \bar{\alpha}
$$

Thus, the space of Dehn invariants for the Mosseri-Sadoc tiles becomes one-dimensional, only the combination $\bar{\alpha}=-\bar{\beta}-\bar{\delta}$ appears.

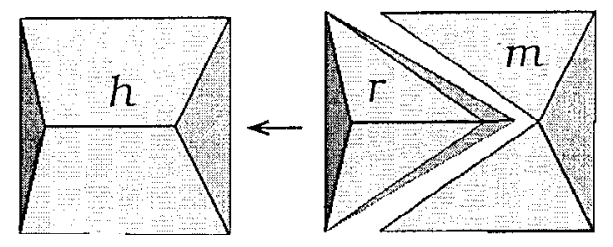

Fig. 3. The tiles $r$ and $m$ always appear together in the projection class of the tilings $\mathcal{T}^{(\mathrm{MS})}$ as the union $h$, $h=r \cup m$. 

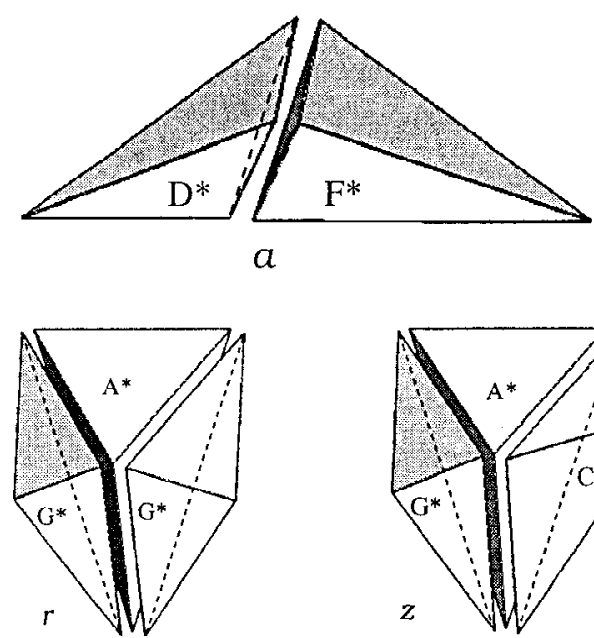
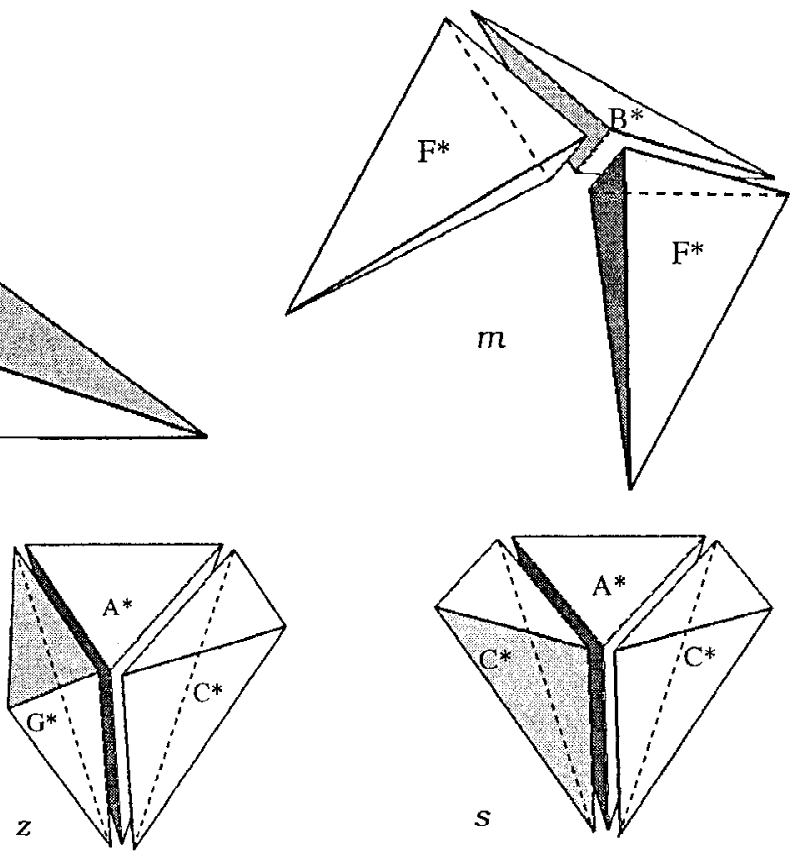

Fig. 4. The tiles $a, m, r, z$, and $s$ are obtained by the packing of the golden tetrahedra.

For the vector of volumes for the Mosseri-Sadoc tiles one obtains

$$
\vec{v}_{\mathrm{MS}}=\operatorname{Vol}\left(\begin{array}{c}
z \\
h \\
s \\
a
\end{array}\right)=\frac{1}{12}\left(\begin{array}{l}
4 \tau+2 \\
6 \tau+4 \\
4 \tau+3 \\
2 \tau+1
\end{array}\right) .
$$

Note. The Mosseri-Sadoc tile $h$ is the union of the tiles $m$ and $r$ introduced in [20]. The volumes and the Dehn invariants of the tiles $m$ and $r$ are

$$
\begin{array}{cc}
\operatorname{Vol}(m)=\frac{1}{12}(2 \tau+3), & \operatorname{Vol}(r)=\frac{1}{12}(4 \tau+1), \\
\mathcal{D}(m)=5(\tau-1) \otimes \bar{\alpha}, & \mathcal{D}(r)=-5(\tau+1) \otimes \bar{\alpha} .
\end{array}
$$

The Dehn invariants of both of them contain only the combination $\bar{\alpha}$. Thus, were the tiles $m$ and $r$ not always glued together, we would not be able to write a matrix equation for the inflation of the five tiles $z, m, r, s$, and $a$. That the tiles $m$ and $r$ in the projection class of the tilings $\mathcal{T}^{\text {(MS) }}$ do always appear together as the prototile $h$ has been shown in [20] by the arguments of the projection method expressed in the "orthogonal space." For an overview of the space tilings obtained by the projection method see [21].

The fact that the Dehn invariants of all five tiles, $z, r, m, s$, and $a$, contain only one angle $\alpha$ has a natural geometric explanation: the faces of all five tiles are composed 
of golden triangles and therefore are orthogonal to the fivefold symmetry axes of the icosahedron.

\subsection{Inflation of Decorated Mosseri-Sadoc Tiles}

Sadoc and Mosseri have given the inflation rules for their $z, h, s$, and $a$ tiles [6]. These rules were for the stone inflation [7] of the tiles. The inflation factor is $\tau=(1+\sqrt{5}) / 2$. The volume inflation matrix of the stone inflation of the tiles is the matrix with integer coefficients. It has been given by Sadoc and Mosseri [6] as

$$
M=\left(\begin{array}{llll}
1 & 1 & 1 & 1 \\
2 & 1 & 2 & 2 \\
1 & 1 & 1 & 2 \\
0 & 0 & 1 & 2
\end{array}\right),
$$

in the following ordering of the tiles: $z, h, s$, and $a$.

In the case of the Mosseri-Sadoc tiles, the stone inflation breaks the symmetry of the tiles. Mosseri and Sadoc [6] have not given a decoration of the tiles which would take care of the symmetry breaking and uniquely define the inflation-deflation procedure at every step. In [20] it has been shown that the projection class of the locally isomorphic tilings $\mathcal{T}^{*(2 F)}$ (see [8]) can be locally transformed into the tilings $\mathcal{T}^{(\mathrm{MS})}, \mathcal{T}^{*(2 F)} \longrightarrow \mathcal{T}^{(\mathrm{MS})}$. The class $\mathcal{T}^{\text {(MS) }}$ of the locally isomorphic tilings of the space by the Mosseri-Sadoc tiles has been defined by the icosahedral projection from the $D_{6}$-lattice [20]. The important property is that minimal packages of the six golden tetrahedra in $\mathcal{T}^{*(2 F)}$, satisfying the condition that their equilateral faces (orthogonal to the threefold directions) are covered, lead to the five tiles $a, s, z, r$, and $m$ [20]. Moreover, the tiles $r$ and $m$ always appear as the union $r \cup m$, that is, tile $h$ of Sadoc and Mosseri with three mirror symmetries [20]. See Figs. 2-4.

It is a priori not evident that the inflation rules for the Mosseri-Sadoc tiles in the projection class of the tilings $\mathcal{T}^{(\mathrm{MS})}$ are the same as those suggested by Sadoc and Mosseri [6].

The inflation rules for the $\mathcal{T}^{*(2 F)}$-tiles in the projection class of the tilings $\mathcal{T}^{*(2 F)}$ have been obtained in [9] and [10]. The inflation rules for the prototiles in a projection class of tilings are determined in the orthogonal space by a procedure explained in [3] and [9]. All edges of the $\mathcal{T}^{*(2 F)}$-tiles carry the arrows and some of these arrows uniquely define the inflation rules for the $\mathcal{T}^{*(2 F)}$-tiles [9]. By a local derivation of $\mathcal{T}^{(\mathrm{MS})}$ from $\mathcal{T}^{*(2 F)}$, the Mosseri-Sadoc tiles inherit these arrows [11]. The arrows which break the symmetry of the $\mathcal{T}^{(\mathrm{MS})}$-tiles define the inflation procedure uniquely. The inflation-deflation rules for the decorated $a, m, r, z$, and $s$ tiles in the projection class of the tilings $\mathcal{T}^{\text {(MS) }}$ are obtained through the local derivation from the inflation-deflation rules for the decorated golden tetrahedra (eight prototiles!) as the tiles of the projection class $\mathcal{T}^{*(2 F)}$. We give the inflation rules for the $a, m, r, z$, and $s$ tiles in Figs. 5-9. If we keep in mind that the tiles $m$ and $r$ appear in $\mathcal{T}^{(\mathrm{MS})}$ together as $h, m \cup r=h$, these are the inflation-deflation rules for the projection class of the tilings $\mathcal{T}^{(\mathrm{MS})}$ of the space by the decorated Mosseri-Sadoc tiles $z, h, s$, and $a$. We see that the inflation rules for $\mathcal{T}^{(\mathrm{MS})}$ as a projection specie [20] are the same (up to the decoration) as for the inflation specie given by Sadoc and Mosseri 


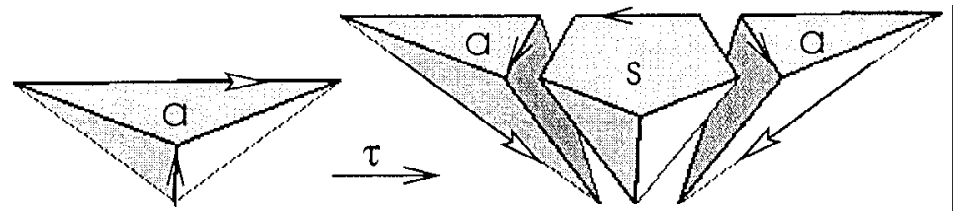

Fig. 5. The inflation rule for the decorated tile $a: \tau a=a \cup s \cup a$. The "white" arrow marks the edge $\tau^{2}(2)$, the "long" edge in the $\tau \mathcal{T}^{*(2 F)}$-class of the tilings (the $\mathcal{T}^{*(2 F)}$-class of the tilings scaled by $\tau$ ).

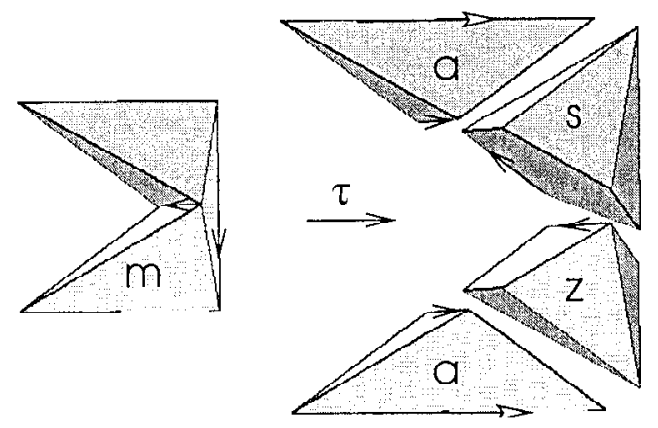

Fig. 6. The inflation rule for the decorated tile $m: \tau m=a \cup s \cup z \cup a$. The white arrow marks the "long" edge in the $\tau \mathcal{T}^{*(2 F)}$-class of tilings.

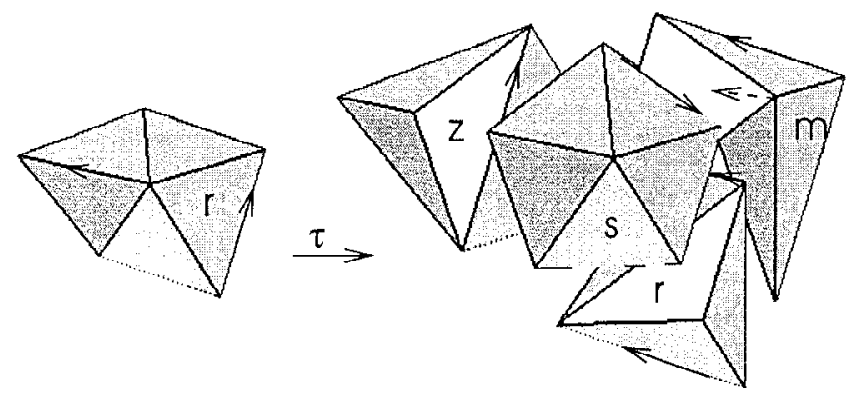

Fig. 7. The inflation rule for the decorated tile $r: \tau r=z \cup s \cup m \cup r$.

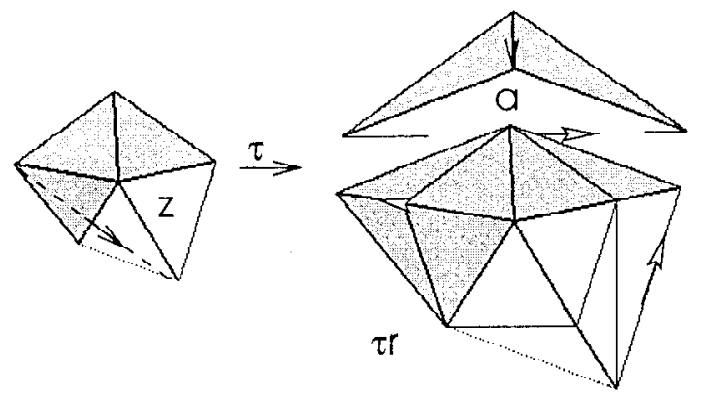

Fig. 8. The inflation rule for the decorated tile $z: \tau z=\tau r \cup a$. The white arrows mark the "short" and the "long" edges in the $\tau \mathcal{T}^{*(2 F)}$-class of tilings. 


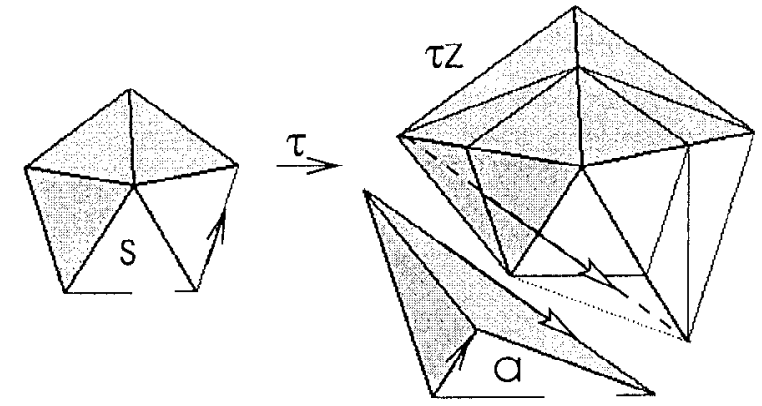

Fig. 9. The inflation rule for the decorated tile $s: \tau s=\tau z \cup a$. The white arrow marks the "long" edge in the $\tau \mathcal{T}^{*(2 F)}$-class of tilings.

[6]. By the fact that only the decorated Mosseri-Sadoc tiles have the uniquely defined inflation-deflation procedure and by the fact that the inflation rules for the projection and inflation species are the same, we identify the inflation [6] and the projection species $[20]$ and denote them by the same symbol, $\mathcal{T}^{(\mathrm{MS})}$.

\subsection{Dehn Invariants and Stone Inflation of Mosseri-Sadoc Tiles}

In this section we show that the inflation matrix for the Mosseri-Sadoc tiles, $z, h, s$, and $a$, can be uniquely reconstructed from the Dehn invariants (and the volume).

Denote the inflation matrix (49) by $M_{\mathrm{MS}}$.

Lemma. A matrix of any stone inflation of the Mosseri-Sadoc tiles must coincide with $M_{\mathrm{MS}}$.

Proof. The vectors $\vec{d}_{\mathrm{MS}}$ and $\vec{v}_{\mathrm{MS}}$ (see (45) and (46)) are eigenvectors of the inflation matrix, with the eigenvalues $\tau$ and $\tau^{3}$ correspondingly (remember that the eigenvalue is equal to the inflation factor to the power which is the dimension of the corresponding invariant).

Explicitly, for the vector of volumes we have

$$
M_{\mathrm{MS}}\left(\begin{array}{c}
4 \tau+2 \\
6 \tau+4 \\
4 \tau+3 \\
2 \tau+1
\end{array}\right)=\left(\begin{array}{c}
16 \tau+10 \\
26 \tau+16 \\
18 \tau+11 \\
8 \tau+5
\end{array}\right)
$$

and for the the vector of Dehn invariants:

$$
M_{\mathrm{MS}}\left(\begin{array}{c}
\tau \\
2 \\
\tau-1 \\
-\tau
\end{array}\right)=\left(\begin{array}{c}
\tau+1 \\
2 \tau \\
1 \\
-\tau-1
\end{array}\right)
$$


As for the golden tetrahedra tiles, assume that the inflation matrix is rational. Then, applying the Galois automorphism, one finds two more eigenvectors of $M_{\mathrm{MS}}$. Again, as for the tetrahedra, this amounts to the decomposition of (50) and (51) in powers of $\tau$.

Together, the four vector equations imply a matrix equation:

$$
M_{\mathrm{MS}}\left(\begin{array}{rrrr}
4 & 2 & 1 & 0 \\
6 & 4 & 0 & 2 \\
4 & 3 & 1 & -1 \\
2 & 1 & -1 & 0
\end{array}\right)=\left(\begin{array}{rrrr}
16 & 10 & 1 & 1 \\
26 & 16 & 2 & 0 \\
18 & 11 & 0 & 1 \\
8 & 5 & -1 & -1
\end{array}\right) .
$$

The solution of this equation is unique and we rediscover the matrix (49).

Note that as for the tetrahedra, the uniqueness happens because of the coincidence: the number of tiles equals the number of invariants times the order of the Galois group.

We stress that even for a stone inflation, the inflation matrix alone is not determining the actual inflation rules of the tiles.

Remarks. 1. The inflation matrix $M_{\mathrm{MS}}$ for the Mosseri-Sadoc tiles is "induced" by the inflation matrix $M_{\mathrm{gt}}$ for the golden tetrahedra in the following sense.

Denote by $V_{\mathrm{gt}}$ a six-dimensional vector space with a basis

$$
\left\{e_{A^{*}}, e_{B^{*}}, e_{C^{*}}, e_{D^{*}}, e_{F^{*}}, e_{G^{*}}\right\}
$$

labeled by the golden tetrahedra. The matrix $M_{\mathrm{gt}}$ acts in the vector space $V_{\mathrm{gt}}$ in an obvious way. We denote the corresponding operator by the same symbol $M_{\mathrm{gt}}$. The lattice $L_{\mathrm{gt}}$ generated by the basis vectors is not preserved by the operator $M_{\mathrm{gt}}$ since the entries of $M_{\mathrm{gt}}$ are not integers.

Denote by $V_{\text {MS }}$ a four-dimensional vector space with a basis

$$
\left\{e_{z}, e_{h}, e_{s}, e_{a}\right\}
$$

labeled by the Mosseri-Sadoc tiles. The basis vectors generate a lattice $L_{\mathrm{MS}}$.

A map $\psi_{\mathrm{gt}}: V_{\mathrm{MS}} \rightarrow V_{\mathrm{gt}}$ given by

$$
\begin{aligned}
& \psi_{\mathrm{gt}}\left(e_{z}\right)=e_{A^{*}}+e_{C^{*}}+e_{G^{*}}, \\
& \psi_{\mathrm{gt}}\left(e_{h}\right)=e_{A^{*}}+e_{B^{*}}+2 e_{F^{*}}+2 e_{G^{*}}, \\
& \psi_{\mathrm{gt}}\left(e_{s}\right)=e_{A^{*}}+2 e_{C^{*}}, \\
& \psi_{\mathrm{gt}}\left(e_{a}\right)=e_{D^{*}}+e_{F^{*}}
\end{aligned}
$$

is an embedding. It is compatible with the lattice structure.

The map $\psi_{\text {gt }}$ reflects the way of packing the golden tetrahedra into the Mosseri-Sadoc tiles (see Fig. 4).

A direct inspection shows that the four-dimensional subspace $\operatorname{Im}\left(\psi_{\mathrm{gt}}\right)$ of $V_{\mathrm{gt}}$ is invariant under the action of $M_{\mathrm{gt}}$ and the matrix of the induced operator in $V_{\mathrm{MS}}$, written in the basis (54), coincides with $M_{\mathrm{MS}}$.

This is quite natural since both matrices, $M_{\mathrm{gt}}$ and $M_{\mathrm{MS}}$, are uniquely determined by the geometrical data-the volumes and the Dehn invariants. 
2. The space $V_{\mathrm{MS}}$ is a subspace in a five-dimensional space $V_{\mathrm{MS}}^{\prime}$ with a basis

$$
\left\{e_{z}, e_{m}, e_{r}, e_{s}, e_{a}\right\}
$$

The element $e_{h}$ is expressed as $e_{h}=e_{m}+e_{r}$.

The space $V_{\mathrm{MS}}^{\prime}$ also maps into $V_{\mathrm{gt}}$, the second line in (55) gets replaced by

$$
\begin{gathered}
\psi_{\mathrm{gt}}\left(e_{m}\right)=e_{B^{*}}+2 e_{F^{*}}, \\
\psi_{\mathrm{gt}}\left(e_{r}\right)=e_{A^{*}}+2 e_{G^{*}} .
\end{gathered}
$$

It is not an embedding any more:

$$
\psi_{\mathrm{gt}}\left(e_{r}+e_{s}\right)=\psi_{\mathrm{gt}}\left(2 e_{z}\right)
$$

This explains again (see (48) and the comment after them) that the inflation matrix for the five tiles $a, m, r, z$, and $s$ cannot be reconstructed from the Dehn invariants and the volumes (in other words, from the matrix $M_{\mathrm{gt}}$ ).

In fact, the inflation matrix for the tiles $a, m, r, z$, and $s$ which reads (in this ordering of the tiles)

$$
\left(\begin{array}{lllll}
2 & 0 & 0 & 0 & 1 \\
2 & 0 & 0 & 1 & 1 \\
0 & 1 & 1 & 1 & 1 \\
1 & 1 & 1 & 1 & 1 \\
2 & 1 & 1 & 1 & 1
\end{array}\right)
$$

is degenerate, so it cannot be induced by the nondegenerate matrix $M_{\mathrm{gt}}$.

3. Denote by $V_{\mathcal{T}^{*(2 F)}}$ an eight-dimensional vector space with a basis

$$
\left\{\tilde{e}_{A^{*}}, \tilde{e}_{B^{*}}, \tilde{e}_{C^{* \mathrm{~b}}}, \tilde{e}_{C^{* \mathrm{r}}}, \tilde{e}_{D^{*}}, \tilde{e}_{F^{*}}, \tilde{e}_{G^{* \mathrm{~b}}}, \tilde{e}_{G^{* \mathrm{r}}}\right\}
$$

labeled by the colored golden tetrahedra. The matrix $M_{\mathcal{T}^{*(2 F)}}$ becomes an operator acting in the space $V_{\mathcal{T}^{*(2 F)}}$.

Define a map $\psi_{\mathcal{T}^{*(2 F)}}: V_{\mathrm{MS}} \rightarrow V_{\mathcal{T}^{*(2 F)}}$ by

$$
\begin{aligned}
& \psi_{\mathcal{T}^{*(2 F)}}\left(e_{z}\right)=\tilde{e}_{A^{*}}+\tilde{e}_{C^{* \mathrm{~b}}}+\tilde{e}_{G^{* \mathrm{r}}}, \\
& \psi_{\mathcal{T}^{*(2 F)}}\left(e_{h}\right)=\tilde{e}_{A^{*}}+\tilde{e}_{B^{*}}+2 \tilde{e}_{F^{*}}+\tilde{e}_{G^{* \mathrm{~b}}}+\tilde{e}_{G^{* \mathrm{r}}}, \\
& \psi_{\mathcal{T}^{*(2 F)}}\left(e_{s}\right)=\tilde{e}_{A^{*}}+\tilde{e}_{C^{* \mathrm{~b}}}+\tilde{e}_{C^{* \mathrm{r}}}, \\
& \psi_{\mathcal{T}^{*(2 F)}}\left(e_{a}\right)=\tilde{e}_{D^{*}}+\tilde{e}_{F^{*}} .
\end{aligned}
$$

The map $\psi_{\mathcal{T}^{*(2 F)}}$ is an embedding.

Again, one can directly check that the subspace $\operatorname{Im}\left(\psi_{\mathcal{T}^{*(2 F)}}\right)$ is invariant under the operator $M_{\mathcal{T}^{*(2 F)}}$ and the matrix of the induced operator in $V_{\mathrm{MS}}$, written in the basis (54), coincides with $M_{\mathrm{MS}}$.

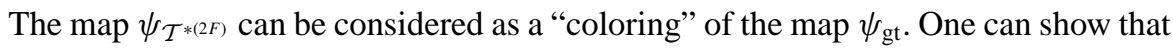
this coloring is unique. 
4. The map $\psi_{\mathcal{T}^{*(2 F)}}$ also extends to the map from the five-dimensional space $V_{\mathrm{MS}}^{\prime}$, the second line in (61) gets replaced by

$$
\begin{aligned}
\psi_{\mathcal{T} *(2 F)}\left(e_{m}\right) & =\tilde{e}_{B^{*}}+2 \tilde{e}_{F^{*}}, \\
\psi_{\mathcal{T}^{*}(2 F)}\left(e_{r}\right) & =\tilde{e}_{A^{*}}+\tilde{e}_{G^{* b}}+\tilde{e}_{G^{* *}} .
\end{aligned}
$$

However, it is still an embedding.

As we have seen in Section 4.1, not only the volume inflation matrix but the actual inflation for the Mosseri-Sadoc tiles (as well as for the five tiles $z, m, r, s$, and $a$ ) is induced by the inflation for $\mathcal{T}^{*(2 F)}$.

\section{Acknowledgments}

It is our pleasure to thank Ludwig Danzer for the careful reading of our manuscript, for the second proof of Lemma 2 in the Section 3.2 and for suggestions of alternative proofs of Lemmas 2 and 3 in Section 3.3. Z. Papadopolos is grateful for the hospitality to the Center of the Theoretical Physics in Marseille, where a part of this work has been done. We also thank the Geometry Center at the University of Minnesota for making Geomview freely available.

\section{Appendix. Geodetic Angles}

In Section 3.2 we showed that the space of Dehn invariants for the golden tetrahedra is two-dimensional. The proof is based on a theorem of Conway et al. [22]. For completeness we briefly remind the reader of the required results from [22].

Definition. An angle $\theta$ is called "pure geodetic" if $\sin ^{2} \theta$ is rational.

Let $\mathcal{E}$ be a vector space spanned over $\mathbb{Q}$ by pure geodetic angles. In [22] a basis of the vector space $\mathcal{E}$ is constructed. It is useful to know the basis: one can check whether some given angles are $\mathbb{Q}$-independent.

An element of the basis of $\mathcal{E}$ is denoted by $\langle p\rangle_{d}$. Here $p$ is a prime integer. The positive integer $d$ has to satisfy two conditions:

1. $d$ is square-free;

2. $(-d)$ is a square modulo $p$.

If $p=2$, then $d \equiv 7(\bmod 8)$ additionally.

To define $\langle p\rangle_{d}$ one solves an equation $4 p^{s}=a^{2}+d b^{2}$ for $a$ and $b$, with the smallest positive $s$. For $d=3$ one requires $b \equiv 0(\bmod 2)$; for $d=1$ one requires $b \equiv 0(\bmod 4)$.

Now,

$$
\langle p\rangle_{d}=\frac{1}{s} \arccos \frac{a}{2 p^{s / 2}} .
$$

Theorem (Conway-Radin-Sadun). The angles $\langle p\rangle_{d}$ together with $\pi$ form a basis in $\mathcal{E}$. 


\section{References}

1. R. Penrose, The role of aesthetics in pure and applied mathematical research, Bull. Inst. Math. Appl. 10 (1974), 266-271.

2. B. Grünbaum and G. C. Shepard, Tilings and Patterns, Freeman, San Francisco, CA, 1987.

3. M. Baake, P. Kramer, M. Schlottmann, and D. Zeidler, Planar patterns with fivefold symmetry as sections of periodic structures in 4-space, Internat. J. Mod. Phys. B4 (1990), 2217-2267.

4. P. Kramer, Non-periodic central space filling with icosahedral symmetry using copies of seven elementary cells, Acta Cryst. Sect. A 38 (1982), 257-264.

5. J. E. S. Socolar and P. J. Steinhardt, Quasicrystals, II: Unit-cell configurations, Phys. Rev. B 34 (1986), 617-647.

6. R. Mosseri and J. F. Sadoc, Two and three dimensional non-periodic networks obtained from self-similar tiling, in The Structure of Non-Crystalline Materials, Taylor and Francis, London, 1982, reprinted in The Physics of Quasicrystals, eds. P. J. Steinhardt and S. Ostlund, World Scientific, Singapore, 1987, pp. 720-734.

7. L. Danzer, Quasiperiodicity; local and global aspects, in Group Theoretical Methods in Physics, eds. V. V. Dodonov and V. I. Man'ko, Lecture Notes in Physics 382, Springer-Verlag, Berlin, 1991, pp. 561-572.

8. P. Kramer, Z. Papadopolos, and D. Zeidler, Symmetries of icosahedral quasicrystals, in Proceedings of the Symposium on Symmetries in Science V: Algebraic Structures, Their Representations, Realizations and Physical Applications, ed. B. Gruber et al., Plenum, New York, 1991, pp. 395-427.

9. Z. Papadopolos, C. Hohneker, and P. Kramer, Tiles-inflation rules for the canonical tilings $\mathcal{T}^{*(2 F)}$ derived by the projection method, Discrete Math. 221(1-3), selected Papers in Honor of Ludwig Danzer, guest eds. E. Schulte and M. Senechal (2000), pp. 101-112.

10. C. Hohneker, P. Kramer, and Z. Papadopolos, Tiles-inflation for the canonical tiling $\mathcal{T}^{*(2 F)}$, in GROUP21 Physical Applications and Mathematical Aspects of Geometry, Groups, and Algebras, Volume 2, eds. H.-D. Doebner, W. Scherer, and C. Schulte, World Scientific, Singapore, 1997, pp. 982-986.

11. Z. Papadopolos and O. Ogievetsky, On inflation rules for Mosseri-Sadoc tilings, Materials Sci. Engrg. A 294-296 (2000), 385-388.

12. D. Hilbert, Mathematische Probleme, Nachr. Gesellschaft Wiss. Göttingen, Math. Phys. (1900), 253-297.

13. M. Dehn, Uber den Rauminhalt, Nachr. Gesellschaft Wiss. Göttingen, Math. Phys. (1900), 345-354; Math. Ann. 55 (1902), 465-478.

14. J. -P. Sydler, Conditions nécessaires et suffisantes pour l'équivalence des polyèdres de l'espace euclidien à trois dimensions, Comment. Math. Helv. 40 (1965), 43-80.

15. V. G. Boltyanski, Hilbert's Third Problem, Nauka, Moscow, 1977 (in Russian).

16. P. McMullen, Nondiscrete regular honeycombs, in Quasicrystals, Networks and Molecules of Fivefold Symmetry, ed. I. Hargittai, VCH, New York, 1990, Chapter 10, pp. 159-179; Quasiperiodic tilings of ordinary space, Internat. J. Mod. Phys. B 7 (1993), 1365-1377.

17. R. Graham, D. Knuth, and O. Patashnik, Concrete Mathematics, Addison-Wesley, Reading, MA, 1989.

18. A. Clark, Elements of Abstract Algebra, Dover, New York, 1971.

19. A. Kurosh, Higher Algebra, Nauka, Moscow, 1946 (in Russian); M. Knebusch and C. Scheiderer, Einführung in die Reelle Algebra, Vieweg Verlag, Braunschweig, 1989.

20. P. Kramer and Z. Papadopolos, The Mosseri-Sadoc tiling derived from the root-lattice $D_{6}$, Canad.J. Phys. 72 (1994), 408-414.

21. Z. Papadopolos and P. Kramer, Models of icosahedral quasicrystals from 6D lattice, Proceedings of the International Conference on Aperiodic Crystals, Aperiodic '94, eds. G. Chapuis et al., World Scientific, Singapore, 1995, pp. 70-76.

22. J. H. Conway, C. Radin, and L. Sadun, On angles whose squared trigonometric functions are rational, Discrete Comput. Geom. 22 (1999), 321-332.

Received October 11, 1999, and in revised form July 5, 2000. Online publication May 4, 2001. 